\title{
THE DISCRETE SCHWARZ-PICK LEMMA FOR OVERLAPPING CIRCLES
}

\author{
JEFF VAN EEUWEN
}

(Communicated by Albert Baernstein II)

\begin{abstract}
Let $P$ and $P^{\prime}$ be circle packings in the hyperbolic plane such that they are combinatorically equivalent, neighboring circles in $P$ overlap one another at some fixed angle between 0 and $\pi / 2$ and the corresponding pairs of circles in $P^{\prime}$ overlap at the same angle, and the radius for any boundary circle of $P$ is less than or equal to that of the corresponding boundary circle of $P^{\prime}$. In this paper we show that the radius of any interior circle of $P$ is less than or equal to that of the corresponding circle in $P^{\prime}$, and the hyperbolic distance between the centers of circles in $P$ is less than or equal to the distance between the corresponding circles in $P^{\prime}$. Furthermore, a single instance of finite equality in either of the above implies equality for all.
\end{abstract}

\section{INTRODUCTION}

In a paper titled The Schwarz-Pick Lemma for circle packings, Beardon and Stephenson [1] prove what they consider a discrete analog for circle packings of the classical Schwarz-Pick Lemma. This result, termed the Discrete SchwarzPick Lemma (DSPL), has been useful in proving other discrete results (e.g., the uniqueness of infinite circle packings of the hyperbolic plane). But the DSPL is restricted in that it only considers circle packings in which neighboring circles are tangent to one another. Because circle packings in which neighboring circles overlap one another at an angle between 0 and $\pi / 2$ (Figure 1 on the next page) have held (see [2]) and continue to hold promise for use in future work, we want to extend the DSPL to include these packings.

It will be assumed that the reader is familiar with the definitions and ideas in [1] and [2]. Many of the proofs given by Beardon and Stephenson in [1] can be extended without much difficulty for the generalized results of this paper. Consequently, many of the proofs and details are left to the reader.

\section{Preliminaries}

Abstract 2-dimensional simplicial complexes are used to represent the combinatorics of a circle packing. We will restrict our attention to complexes, $K$, that are isomorphic to a finite triangulation of a closed topological disc and have

Received by the editors August 12, 1992 and, in revised form, November 2, 1992.

1991 Mathematics Subject Classification. Primary 30C80; Secondary 51M99, 31C20.

The author gratefully acknowledges support of the Tennessee Science Alliance. 


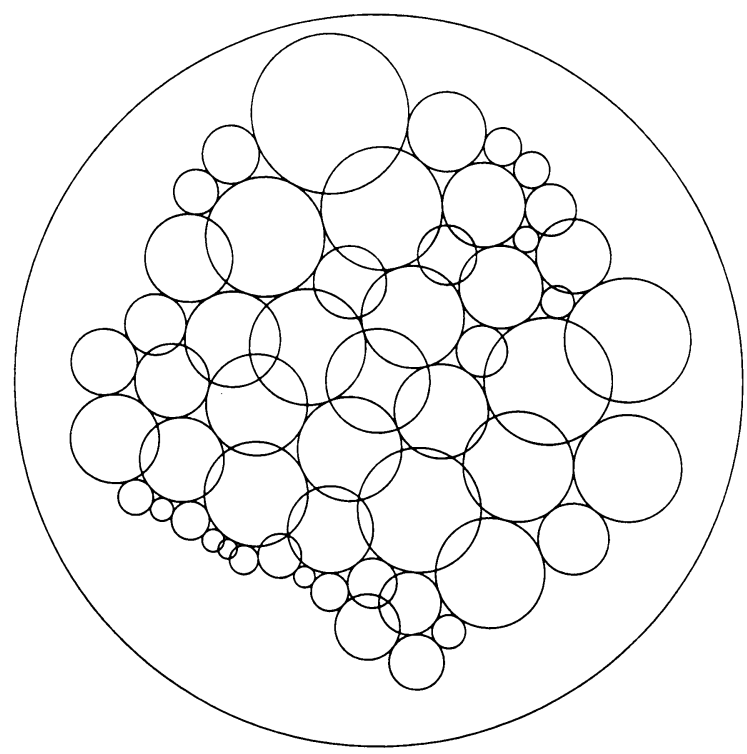

FIGURE 1

the following properties: every boundary vertex shares an edge with at least one interior vertex, and any pair of interior vertices can be connected by an edge path whose edges have only interior vertices as end points. Complexes of this type will be termed special.

Let $V$ and $E$ be the set of vertices and edges of $K$ respectively. A function $R: V \rightarrow(0, \infty]$ will be called a label for $K$. It will be assumed that $R$ is finite on interior vertices. A function $\varphi: E \rightarrow[0, \pi / 2]$ will be called an angle assignment for $K$ if it satisfies the following:

(i) if $e_{1}, e_{2}, e_{3} \in E$ are distinct and form a closed edge path and $\varphi\left(e_{1}\right)+$ $\varphi\left(e_{2}\right)+\varphi\left(e_{3}\right) \geq \pi$, then $e_{1}, e_{2}, e_{3}$ are the edges of a face of $K$,

(ii) if $e_{1}, e_{2}, e_{3}, e_{4}$ are distinct and form a closed edge path and $\varphi\left(e_{i}\right)=$ $\pi / 2, i=1,2,3,4$, then $e_{1}, e_{2}, e_{3}, e_{4}$ are the edges of two faces that share a common edge $e$.

A hyperbolic structure can be placed onto the complex $K$ in the following manner. Let $R$ and $\varphi$ be given. Then to each face of $K$ there corresponds a triple of radii and overlap angles. From these triples come three circles in the hyperbolic plane having the given radii and overlap angles. The hyperbolic triangle formed by joining the centers of these circles is associated to the face of $K$ pointwise so that corresponding vertices are identified, thus inducing a hyperbolic structure onto this face. If two faces are contiguous, then their associated triangles can be made to be contiguous. By adjusting the identifications of those two faces, the hyperbolic structure is made compatible across the shared edge. The complex $K$ along with the hyperbolic structure determined by $R$ and $\varphi$ is called a hyperbolic complex and will be denoted by $K(R, \varphi)$.

With the hyperbolic structure on $K$, each face of $K$ has the structure of a hyperbolic triangle. At a given vertex of $K$, every face in the star of the given vertex forms an angle at this vertex. The sum of all these angles will be termed the angle sum at the vertex. We will call $K(R, \varphi)$ a packing if the angle sum 
at every interior vertex is equal to $2 \pi . K(R, \varphi)$ will be called a subpacking if the angle sum at every interior vertex is greater than or equal to $2 \pi$.

The hyperbolic structure on $K(R, \varphi)$ is compatible across shared edges, but it may or may not be at shared vertices. If $\varphi$ is constantly 0 , there is a discussion in [1] of when the hyperbolic structure on $K(R, \varphi)$ is compatible at shared vertices and how it compares both locally and globally to the hyperbolic plane. This discussion can be extended to include any angle assignment $\varphi$.

\section{MoNOTONICITY RESULTS}

We need to establish a collection of monotonicity results that will be essential in the proof of our main result. Refer to Figure 2 for

Lemma 1. Let $r_{0}, r_{1}, r_{2} \in(0, \infty]$ be the radii for the circles with centers $z_{0}, z_{1}$, $z_{2}$ and overlap angles $\alpha_{01}, \alpha_{02}, \alpha_{12} \in[0, \pi / 2]$. Then:

(i) $\alpha\left(r_{0}\right)$ is a continuous strictly decreasing function.

(ii) $\alpha\left(r_{0}\right)$ takes on every value in $\left[0, \pi-\alpha_{1,2}\right)$ with $\alpha\left(r_{0}\right) \rightarrow \pi-\alpha_{12}$ as $r_{0} \rightarrow 0$.

(iii) $\beta\left(r_{0}\right),\left(\gamma\left(r_{0}\right)\right)$ is a continuous strictly increasing function when $r_{1}<\infty$ $\left(r_{2}<\infty\right)$, except if $\alpha_{01}=0$ and $\alpha_{02}=\alpha_{12}=\pi / 2, \quad\left(\alpha_{02}=0, \alpha_{01}=\right.$ $\left.\alpha_{12}=\pi / 2\right)$, in which case it is constant.

(iv) The area of the triangle of centers is a strictly increasing function of $r_{0}$.

(v) Let $p$ be a point on the edge of the triangle of centers opposite $z_{0}$. Let $l_{P}\left(r_{0}\right)$ denote the distance from $p$ to $z_{0}$. If at most one of $r_{1}, r_{2}$ is infinite then $d l_{P} / d r_{0}$ is positive and continuous.

Furthermore, if both $r_{1}$ and $r_{2}$ are finite then there exists a continuous function, $\tau\left(r_{0}\right)$, such that for any $p, d l_{P} / d r_{0}>\tau\left(r_{0}\right)>0$.

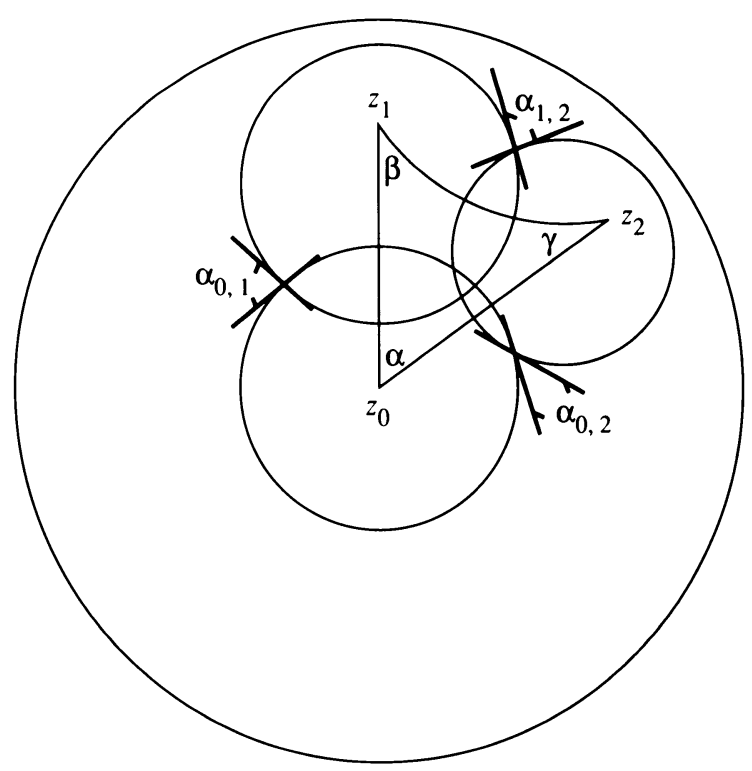

FIGURE 2. Triangle of centers and notation 
An angle assignment $\varphi$ will be called a strict angle assignment if $\varphi(e) \neq 0$ for any edge $e$ such that $e$ is the common edge of two contiguous faces where $\varphi$ is $\pi / 2$ on the remaining four edges. From Lemma 1 we get

Lemma 2. Let $\nu$ be a vertex of $K(R, \varphi)$ with neighboring vertices $\nu_{1}, \ldots, \nu_{n}$. Let $r, 0<r<\infty$, and $r_{1}, \ldots, r_{n}, 0<i_{i} \leq \infty$, denote their radii respectively. Let $\theta=\theta\left(r, r_{1}, \ldots, r_{n}\right)$ be the resulting angle sum at $\nu$. Then:

(i) $\theta$ is continuous and strictly decreasing in $r$.

(ii) $\theta$ is continuous and increasing in $r_{i}, 1 \leq i \leq n$.

(iii) Fix $r_{1}, \ldots, r_{n}$. Given any $\omega, 0 \leq \omega<\sum\left(\pi-\alpha_{i j}\right)$, there exist a unique radius $r^{\prime}>0$ for which $\theta\left(r^{\prime}, r_{1}, \ldots, r_{n}\right)=\omega$.

Furthermore, if $\varphi$ is a strict angle assignment then monotonicity is strict in (ii).

Given $R$ and $\varphi$, define the metric, $\rho_{R}($,$) , on K(R, \varphi)$ to be the infemum of the lengths of paths between the two points in $K(R, \varphi)$. The notation $\rho_{R}($,$) does not show that this metric is dependent on \varphi$. This is because interest lies in what happens to $\rho_{R}($,$) as the label is changed and \varphi$ remains fixed.

Lemma 3. Let $\varphi$ be fixed. If $K(R, \varphi)$ and $K\left(R^{\prime}, \varphi\right)$ are hyperbolic complexes such that $R^{\prime}(\nu) \leq R(\nu)$ for every vertex $\nu$ then $\rho_{R^{\prime}}\left(\nu_{1}, \nu_{2}\right) \leq \rho_{R}\left(\nu_{1}, \nu_{2}\right)$ for every pair of vertices. Also if $\nu_{1}$ satisfies $R^{\prime}\left(\nu_{1}\right)<R\left(\nu_{1}\right)$ and $\rho_{R}\left(\nu_{1}, \nu_{2}\right)<\infty$ then the inequality is strict.

The proof consists of starting with a path in $K(R, \varphi)$ between $v_{1}$ and $v_{2}$ and showing the existence of a shorter path in $K\left(R^{\prime}, \varphi\right)$. This is accomplished by taking advantage of Lemma $1(v)$.

We want to find an upper bound on the radius at an interior vertex $v$ of $K(R, \varphi)$ with neighbors $v_{1}, \ldots, v_{n}$. To do this, observe (refer to Figure 2)

(i) If $0<r_{0}<\infty$ and $0<r_{1}, r_{2} \leq \infty$ then $\alpha$ is a decreasing function of $\alpha_{12}$ on $[0, \pi / 2]$.

(ii) If $0<r_{0}<\infty$ and $0<r_{1}, r_{2} \leq \infty$ then $\gamma$ is an increasing function $\alpha_{12}$ on $[0, \pi / 2]$.

(iii) When $\alpha_{01}=\alpha_{02}=\pi / 2$ and $\alpha_{12}=0$, the circle centered at $z_{0}$ intersects the other two circles at their point of tangency.

Lemma 4. Let $\nu$ be an interior vertex of $K(R, \varphi)$ with neighbors $\nu_{1}, \ldots, \nu_{n}$. Let $R(\nu)=r<\infty$ and $R\left(\nu_{i}\right)=r_{i}$. If the angle sum $\theta(\nu)$ at $\nu$ is greater than or equal to $2 \pi$ then $r<\operatorname{sqrt}(n)$.

Proof. By observations (i) and (ii), decreasing the overlap angles between all pairs of neighboring circles having vertices in $\left\{v_{1}, \ldots, v_{n}\right\}$ to zero and increasing the overlap angles between the pairs of circles having vertices $v_{i}$ and $v$ to $\pi / 2$ will increase $\theta$. By Lemma 2 increasing each $r_{i}$ to $\infty$ will again increase $\theta$. By Lemma 2(iii) $r$ can be increased to some $r^{\prime}$ until $\theta=2 \pi$. Let $P$ be the polygon formed by connecting neighboring $v_{i}$ 's with geodesics. By observation (iii) the circle of radius $r^{\prime}$ is contained in $P$. Comparing the area of the circle with that of the polygon, we have that

$$
\pi\left(r^{\prime}\right)^{2} \leq 4 \pi\left(r^{\prime} / 2\right)^{2} \leq 4 \pi \sinh ^{2}\left(r^{\prime} / 2\right) \leq(n-2) \pi .
$$




\section{The Discrete Schwarz-Pick Lemma for overlapping Circles}

Let $K, \varphi$, and boundary radii for $K$ be given. Define $\Re$ to be the set of all labels $R$ such that $K(R, \varphi)$ is a subpacking and has boundary radii less than or equal to the given boundary radii. By the Andreev-Thurston Theorem we have that $\mathfrak{R}$ is nonempty. Define $\sup \mathfrak{R}(v)=\sup \{R(v): R \in \mathfrak{R}\}$ for every vertex $v$ of $K$. Using the monotonicity results of Lemma 2 and the bound for interior radii given in Lemma 4 , it is not hard to see that $\sup \mathfrak{R}$ is the unique packing label for $K$ having the prescribed boundary radii. We are now in a position to prove a generalization of the DSPL.

Theorem 1. Let $K$ be a special complex and let $\varphi$ be an angle assignment for $K$. If $R$ and $R_{P}$ are labels for $K$ such that $K(R, \varphi)$ is a subpacking, $K\left(R_{P}, \varphi\right)$ is a packing, and $R(\nu) \leq R_{P}(\nu)$ for every boundary vertex $\nu$, then

(i) $R(\nu) \leq R_{P}(\nu)$ for every vertex $\nu$,

(ii) $\rho_{R}\left(\nu_{1}, \nu_{2}\right) \leq \rho_{R_{P}}\left(\nu_{1}, \nu_{2}\right)$ for any pair of vertices in $K$, and

(iii) $\operatorname{Area}_{R}(f) \leq \operatorname{Area}_{R_{P}}(f)$ for every face $f$ of $K$.

Furthermore, if $\varphi$ is a strict angle assignment and equality holds for either a single interior vertex in (i), a single pair of interior vertices in (ii), or a single face in (iii) then $R=R_{P}$.

Proof. (i) The previous discussion gives this result. If $\varphi$ is a strict angle assignment and equality holds at a single interior vertex $v_{0}$ then we claim it holds for all the neighboring vertices. Suppose that there exists a neighbor $w$ such that $R(w)<R_{P}(w)$. Since the angle sum at $v_{0}$ is an increasing function of the radius at $w$ and $\theta_{R}\left(v_{0}\right) \geq 2 \pi, \theta_{R_{P}}\left(v_{0}\right)>2 \pi$. This contradicts that $R_{P}$ is a packing label. Hence there must be equality at all of $v_{0}$ 's neighbors. This equality spreads to all vertices by repeating the above argument.

(ii) Lemma 3 and (i) give this result. The case of equality comes from the last part of Lemma 3 and (i).

(iii) Every face has at least one interior vertex $v$. This and Lemma 1(iv) give the inequality. Since monotonicity is strict, the area of two faces being equal implies $R(v)=R_{P}(v)$. By (i), $R=R_{P}$.

Simple examples show that $\varphi$ needs to be a strict angle assignment in the case of equality.

\section{REFERENCES}

1. Alan F. Beardon and Kenneth R. Stephenson, The Schwarz-Pick Lemma for circle packings, Illinois J. Math. 35 (1991), 577-606.

2. William Thurston, The geometry and topology of 3-manifolds, preprint, Princeton University Notes.

Department of Mathematics, University of Tennessee, Knoxville, Tennessee 37996

Current address: Department of Mathematics, Michigan State University, East Lansing, Michigan 48824 